\title{
Processamento mínimo de tubérculos de batata de baixo valor comercial
}

\author{
Marlene T Lovattoㅜ; Dilson A Bisognin ${ }^{1 *}$; Rosa de O Treptow ${ }^{2}$; Lindolfo Storck ${ }^{1}$; Francisco S Gnocato ${ }^{1}$; \\ Glademir Morin Junior ${ }^{1}$ \\ ${ }^{1}$ UFSM-CCR, Depto. Fitotecnia, 97105-900 Santa Maria-RS; mtlovatto@gmail.com; dilsonb@smail.ufsm.br (*autor para correspondên- \\ cia); lindolfo@pq.cnpq.br; fgnocato@gmail.com; glademirmorin@gmail.com; ${ }^{2}$ Embrapa Clima Temperado, C. Postal 403, 96001-970 \\ Pelotas-RS; rotreptow@hotmail.com
}

\begin{abstract}
RESUMO
Foram testados diferentes métodos para controlar o escurecimento de tubérculos de batata de baixo valor comercial para o mercado fresco e prolongar a vida de prateleira do produto minimamente processado. Uma mistura de tubérculos de duas cultivares de batata com diâmetro entre 30 e $45 \mathrm{~mm}$, de baixo valor comercial para o mercado fresco, foram minimamente processados, submetidos a tratamentos à base de metabissulfito de sódio, ácido ascórbico e branqueamento, embalados e armazenados a $5^{\circ} \mathrm{C}$. Os tubérculos minimamente processados foram avaliados quanto ao $\mathrm{pH}$, acidez titulável, teor de vitamina C, cor, aparência, textura, sabor e intenção de compra aos $0,5,10$ e 15 dias de armazenamento. Os valores intermediários e aceitáveis para os atributos aparência, sabor e textura eram esperados e estão conformes com a matéria-prima utilizada. Tubérculos de batata de baixo valor comercial para o mercado fresco podem ser aproveitados para a elaboração de produtos minimamente processados, com boa aceitação até o quinto dia de armazenamento a $5^{\circ} \mathrm{C}$. A manutenção da vida de prateleira dos tubérculos minimamente processados de batata depende da aplicação do metabissulfito de sódio.
\end{abstract}

Palavras-chave: Solanum tuberosum, metabissulfito de sódio, branqueamento, ácido ascórbico, análise sensorial, produtos inovadores.

\begin{abstract}
Minimal processing of potato tubers with low commercial value

Different methods of browning control were evaluated for potato tubers with low commercial value to the fresh market and to increase the shelf life period of the minimal processed product. A tuber blend of two potato cultivars, with diameters between 30 and $45 \mathrm{~mm}$ and low commercial value to the fresh market, was minimally processed. Tubers were immersed in the treatment solutions of sodium metabisulfite, ascorbic acid and bleaching. Treated tubers were packaged and stored at $5^{\circ} \mathrm{C}$ for evaluations. The evaluations were $\mathrm{pH}$, titratable acidity, vitamin $\mathrm{C}$, color, appearance, texture, taste and purchase intention at $0,5,10$, and 15 days of storage. The results of appearance, flavor and texture varied from acceptable to intermediate values and they were in agreement with the heterogeneity of the processed tubers. Potato tubers with low commercial value to the fresh market are suitable for the elaboration of minimal processed products of good acceptance until the fifth day of storage at $5^{\circ} \mathrm{C}$. The treatment with sodium metabisulfite is required to keep a desirable shelf life period.
\end{abstract}

Keywords: Solanum tuberosum, sodium metabisulfite, bleaching, ascorbic acid, sensorial analysis, innovative products.

(Recebido para publicação em 15 de abril de 2011; aceito em 28 de maio de 2012) (Received on April 15, 2011; accepted on May 28, 2012)

U $\mathrm{m}$ dos fatores que transformou a batata (Solanum tuberosum) na hortaliça mais apreciada, reverenciada e consumida no mundo foi a facilidade de preparo. O cozimento pode ser realizado na gordura, na água, no vapor ou no forno, para ser prontamente consumida (Haase, 2008). Do ponto de vista da segurança alimentar, a batata é uma cultura com alta capacidade de produzir carboidratos, proteínas, vitaminas e minerais em curto período de tempo (Gray \& Hughes, 1978). Duzentos gramas de batata cozida fornecem $6 \%$ da energia, $0,3 \%$ da gordura, 11\% dos carboidratos, 9\% da proteína, $11 \%$ da fibra, $28 \%$ do potássio, $47 \%$ da vitamina $\mathrm{C}$ e $8 \%$ da vitamina $\mathrm{B}_{2}$ do requerimento diário recomendado para uma pessoa (Haase, 2008). Além do alto valor nutricional e da importância para a economia nacional, a batata pode ser acrescentada na lista de alimentos minimamente processados, pois alia conveniência e qualidade de produto fresco (Cabezas-Serrano et al., 2009), com disponibilidade durante todo o ano (Fonseca, 2007).

O processo de industrialização é visto como uma possibilidade para agregação de valor aos produtos primários (Endo et al., 2006) e redução de desperdícios (Pilon, 2003; Endo et al., 2006). No Brasil, entre 6 e 7\% dos tubérculos de batata produzidos são processados antes da comercialização, principalmente na forma de batata palha (Popp, 2005). Dos tubérculos colhidos, 20 a $50 \%$ pertencem às classes primeirinha e diversas (diâmetro entre 30 e $45 \mathrm{~mm}$ ), comercializadas por um preço 40 a 50\% inferior ao do tamanho classificado como extra, que é a classe mais aceita pelo consumidor (Pineli \& Moreti, 2004). Esses tubérculos de baixo valor comercial para o mercado fresco podem ser aproveitados para elaborar produtos minimamente processados, o que agregaria valor e aumentaria o retorno econômico e a sustentabilidade econômica da cadeia produtiva da batata (Nantes \& Leonelli, 2000).

Os produtos minimamente processados podem ser definidos como frutas ou vegetais, ou combinação desses, que foram descascados, picados, torneados ou ralados, dentre outros processos, mas que mantenham a qualidade e o fres- 
cor (Moretti, 2007). O processamento promove o aumento da respiração e da produção de etileno e a indução e síntese de compostos fenólicos para a cicatrização dos tecidos (Moretti, 2007). Além disso, acarreta o extravasamento de substratos fenólicos do vacúolo, que entram em contato com as enzimas catalisadoras das reações de oxidação dos polifenóis (polifenoloxidase) presentes no citoplasma, formando as melaninas (Alencar \& Koblitz, 2008), que resultam no escurecimento enzimático (Jacomino et al., 2008). Batatas descascadas ou cortadas são extremamente sensíveis ao escurecimento enzimático (Rocha et al., 2003), que é um problema para indústria de alimentos e pode afetar a aparência e as propriedades organolépticas (Rocculi et al., 2007), resultando na diminuição da vida de prateleira e do valor de mercado (Ahvenainen, 1996).

Diversas substâncias químicas, em diferentes combinações e concentrações, são eficazes para inibir as reações de escurecimento (Laurila et al., 1998). As mais utilizadas são os sulfitos e os ácidos, pelo baixo custo, facilidade na aplicação e efetividade do tratamento (Araújo, 2010). A manutenção da vida de prateleira dos produtos minimamente processados também depende da descontaminação microbiana (Gómez-López et al., 2009) e do uso de embalagens adequadas e de refrigeração (Rocculi et al., 2007). Na Europa, a vida de prateleira varia de 5 a 7 dias, enquanto que nos Estados Unidos varia de 10 a 14 dias (Jaouen, 2006).

O objetivo deste trabalho foi testar diferentes métodos para controlar o escurecimento de tubérculos de batata de baixo valor comercial para o mercado fresco e prolongar a vida de prateleira do produto minimamente processado.

\section{MATERIAL E MÉTODOS}

O experimento foi conduzido em julho de 2009, na Universidade Federal de Santa Maria. A matéria-prima foi constituída por uma mistura de tubérculos de batata colhidos em junho, das cultivares Macaca e Asterix, numa proporção de $50 \%$, com formatos característicos de cada cultivar (respectivamente arredondado e alongado) e menor diâmetro entre
30 e $45 \mathrm{~mm}$, por ser uma matéria-prima de baixo valor comercial para o mercado fresco e representativa do processo de classificação, lavagem e distribuição de tubérculos de batata.

Os tubérculos foram lavados em água potável e sanitizados em hipoclorito de sódio na concentração de 200 mg L $\mathrm{L}^{-1}$ de cloro livre e $\mathrm{pH} \mathrm{7,0}$ por 15 min. Os tubérculos foram então descascados mecanicamente e aparados (remoção manual das imperfeições, restos de casca e manchas escuras). A seguir foram sanitizados em hipoclorito de sódio na concentração de $100 \mathrm{mg}$ $\mathrm{L}^{-1}$ de cloro livre e $\mathrm{pH}$ 7,0 por $10 \mathrm{~min}$. Após, foram centrifugados a $460 \mathrm{rpm}$ por $4 \mathrm{~min}$. e imediatamente imersos nas respectivas soluções de tratamento por $10 \mathrm{~min}$. e novamente centrifugados antes do acondicionamento. Os tratamentos utilizados foram: 1) água potável (controle); 2) metabissulfito de sódio a $0,1 \%$; 3) metabissulfito de sódio a $0,2 \% ; 4)$ metabissulfito de sódio a $0,1 \%+$ ácido ascórbico a $1 \%$; 5) branqueamento + metabissulfito de sódio a $0,1 \% ; 6$ ) branqueamento + metabissulfito de sódio a $0,1 \%$ com vácuo. O branqueamento foi realizado pela imersão dos tubérculos em água potável a $80^{\circ} \mathrm{C}$, por $5 \mathrm{~min}$. Todas as amostras, com aproximadamente $600 \mathrm{~g}$ de tubérculos, foram acondicionadas em embalagens de $17 \times 25 \mathrm{~cm}$, composta por cinco camadas de material coextrusado, e seladas em empacotadora comercial. As embalagens do tratamento com vácuo foram submetidas à pressão de $540 \mathrm{~mm} \mathrm{Hg}$ por $20 \mathrm{~s}$. O fluxograma de preparação de batatas minimamente processadas e os tratamentos utilizados para o controle do escurecimento e a manutenção da vida de prateleira foram definidos em uma série de experimentos preliminares realizados nas mesmas condições experimentais durante o ano de 2008.

As amostras foram armazenadas à temperatura de $5 \pm 1^{\circ} \mathrm{C}$ e avaliadas aos $0,5,10$ e 15 dias. Foram determinados o pH (Instituto Adolfo Lutz, 2009), a acidez titulável (\% de ácido cítrico) (AOAC, 2005), a cor no sistema de coordenadas $L * a * b *$ (Minolta CR 310) e o teor de vitamina $\mathrm{C}$ pelo teor de ácido ascórbico (Jacques-Silva et al., 2001). A qualidade foi avaliada pela técnica de análise descritiva quantitativa (Queiroz \& Treptow, 2006) com a utilização de nove julgadores treinados. Foram analisados os atributos aparência (formato, tamanho, cor, defeitos e qualidade geral), textura (dureza na primeira mordida, cremosidade e umidade), sabor (amargo, sabor característico, sabor estranho) e intenção de compra.

O experimento com seis tratamentos de processamento mínimo foi conduzido no delineamento inteiramente casualizado com nove repetições e avaliado em quatro tempos de armazenamento $(0,5,10$ e 15 dias). Os dados médios dos quatro tempos do caráter cor foram submetidos à análise da variância e teste $\mathrm{F}$, sendo as médias comparadas pelo teste de Duncan $(\mathrm{p}=0,05)$. Para os demais caracteres, os tratamentos de processamento mínimo, para cada tempo de armazenamento, foram submetidos ao teste de Kruskal-Wallis que, sendo significativo $(p=0,05)$, foram procedidas as comparações múltiplas de Kruskal-Wallis $(\mathrm{p}=0,05)$. Também, para cada tratamento, foi aplicado o teste de Kruskal-Wallis e comparações múltiplas de Kruskal-Wallis para a comparação dos tempos de armazenamento. As análises foram realizadas com o auxílio do aplicativo computacional SAEG (2007).

\section{RESULTADOS E DISCUSSÃO}

Os valores de $\mathrm{pH}$ e acidez titulável e o teor de vitamina $\mathrm{C}$, medido pelo teor de ácido ascórbico, pouco variaram entre os tratamentos e o período de armazenamento (dados não apresentados). $\mathrm{O}$ pH dos tubérculos minimamente processados apresentou uma leve redução em função do armazenamento, cujas diferenças foram detectadas a partir dos 10 dias, porém manteve-se entre 5,0 e 7,0, conforme preconizados por Carvalho \& Castro (2002). Os valores de acidez titulável foram menores nos tratamentos com o branqueamento $(0,10$ a 0,20$)$, devido à utilização do ácido clorogênico na formação do complexo com o íon ferroso (Friedman, 1997). Os valores médios aumentaram durante o armazenamento $(0,18$ a 0,23$)$, cujo aumento foi mais discreto nos tratamentos com o branqueamento. O teor de vitamina $\mathrm{C}$ variou com os tratamentos entre 
Tabela 1. Cor medida pelas coordenadas $L * a * b *$ dos tubérculos de batata submetidos a tratamentos de processamento mínimo na média das quatro avaliações durante o armazenamento a $5 \pm 1^{\circ} \mathrm{C}$ por 15 dias [color coordinates measured by $\mathrm{L} * \mathrm{a} * \mathrm{~b} *$ of potato tubers submitted to minimal processing treatments on the average of four evaluations during storage at $5+1{ }^{\circ} \mathrm{C}$ for 15 days]. Santa Maria, UFSM, 2010.

\begin{tabular}{|c|c|c|c|c|c|c|}
\hline \multirow{2}{*}{ Tratamentos } & \multicolumn{6}{|c|}{ Coordenadas de cor } \\
\hline & $L^{*}$ & & $a^{*}$ & & $b^{*}$ & \\
\hline Água potável (controle) & 44,75 & $b^{2}$ & 2,26 & $\mathrm{a}$ & 15,00 & $\mathrm{~d}$ \\
\hline Metabis. sódio $(0,1 \%)$ & 56,24 & a & $-0,55$ & $\mathrm{~b}$ & 24,22 & $\mathrm{a}$ \\
\hline Metabis. sódio $(0,2 \%)$ & 57,35 & $\mathrm{a}$ & $-0,68$ & $\mathrm{~b}$ & 24,62 & A \\
\hline Metabis. sódio $(0,1 \%)+$ ác. ascórbico $(1 \%)$ & 56,70 & $\mathrm{a}$ & $-1,17$ & $\mathrm{c}$ & 22,46 & B \\
\hline Branq $^{1} .+$ metabis. sódio $(0,1 \%)$ & 56,86 & $\mathrm{a}$ & $-2,51$ & $\mathrm{~d}$ & 20,58 & $\mathrm{C}$ \\
\hline Branq. + metabis. sódio $(0,1 \%)$ com vácuo & 55,31 & $\mathrm{a}$ & $-2,90$ & d & 21,62 & $\mathrm{BC}$ \\
\hline
\end{tabular}

${ }^{1}$ Branqueamento em água aquecida a $80^{\circ} \mathrm{C}$ (proporção de $1 \mathrm{~kg}$ de amostra para $2 \mathrm{~L}$ de água) por $5 \mathrm{~min}$ [blanching in water heated at $80^{\circ} \mathrm{C}$ (ratio of $1 \mathrm{~kg}$ of sample in $2 \mathrm{~L}$ of water) during $5 \mathrm{~min}$ ]; ${ }^{2}$ Tratamentos seguidos por letras diferentes na coluna diferem entre si pelo teste de Duncan, a $5 \%$ de probabilidade de erro (treatments followed by different letters in the column differ by Duncan test at $5 \%$ of probability).

9,10 e $27,44 \mathrm{mg} 100 \mathrm{~g}^{-1}$. Os maiores teores de vitamina $\mathrm{C}$ foram detectados no tratamento com branqueamento + metabissulfito de sódio com vácuo.

Os resultados obtidos com $\mathrm{pH}$ estão de acordo com os de Gregory (2010) que afirma que a diminuição do $\mathrm{pH}$, devido a efeitos térmicos, pode favorecer a estabilidade de vitaminas, como a vitamina $\mathrm{C}$, e ajuda a evitar o escurecimento enzimático e a diminuir a carga microbiana (Alencar \& Koblitz, 2008). A polpa crua de batata possui aproximadamente $17,4 \mathrm{mg} 100 \mathrm{~g}^{-1} \mathrm{de}$ vitamina $\mathrm{C}$ e, após o cozimento, esse valor é reduzido para $13,1 \mathrm{mg} 100 \mathrm{~g}^{-1}$ (Chitarra \& Chitarra, 2005). Neste estudo foi avaliado o branqueamento que não afetou o teor de vitamina $\mathrm{C}$. No entanto, o teor de vitamina $\mathrm{C}$ aumentou com o armazenamento, o que está de acordo com o observado por Tudela et al. (2002) em tubérculos descascados e armazenados a $4^{\circ} \mathrm{C}$ de todas as cultivares estudadas.

Tendo em vista que a maior variação de cor dos tubérculos ocorreu entre os tratamentos para o controle do escurecimento dos tubérculos minimamente processados e que o armazenamento pouco afetou a cor dos tubérculos, os resultados apresentados se referem a uma média das quatro avaliações de cor durante o armazenamento (Tabela 1). Conforme esperado, o maior escurecimento foi verificado no controle (menor valor de $L^{*}$ ), indicando que todos os tratamentos aplicados foram efetivos para inibir o escurecimento. Para o valor de $a^{*}$ pode-se verificar uma coloração avermelhada no controle, comprovando a ocorrência do escurecimento, que tem início com a coloração avermelhada, enquanto que nos tratamentos com apenas metabissulfito os tubérculos permaneceram com uma coloração intermediária entre o vermelho e verde. Esses resultados mostram o efeito do metabissulfito em inibir o escurecimento, que foi potencializado pela combinação com ácido ascórbico. Nos tratamentos com branqueamento ocorreu uma coloração acinzentada, provavelmente atribuída à formação de um complexo entre o ácido clorogênico e o íon ferro, que desenvolve a coloração cinza azulada (Friedman, 1997). Portanto, a aplicação de branqueamento promove a formação de cor não característica de tubérculos recém descascados, o que poderia causar a rejeição da batata minimamente processada pelos consumidores.

A análise de aparência dos tubérculos de batata minimamente processados mostrou uma grande variação para tamanho, formato, defeitos (manchas), cor e qualidade geral (Tabela 2). Essa variação era esperada, devido a heterogeneidade dos tubérculos utilizados, o que é um desafio para a obtenção da matéria prima. A padronização da aparência do produto minimamente processado é um desafio geral para a indústria na melhoria da qualidade sensorial e na eliminação de defeitos externos e de superfícies endurecidas (Thybo et al., 2006). A utilização de tubérculos de uma única cultivar já representaria uma grande melhoria da qualidade geral dos produtos minimamente processados, devido à padronização da aparência, e poderia ser implementada com alguma facilidade pelas empresas de classificação, lavagem e distribuição de batata, tendo em vista o potencial de agregação de valor.

Durante o período de armazenamento, os tubérculos submetidos aos tratamentos somente com metabissulfito de sódio e combinado com ácido ascórbico, foram superiores na qualidade geral. Os tratamentos com branqueamento causaram a retrogradação do amido (Lante \& Zocca, 2010), resultando na liberação de líquidos dentro da embalagem e no desenvolvimento de coloração acinzentada, provocada pela reação entre o ácido clorogênico e o íon ferro (Friedman, 1997).

Para o atributo sabor houve diferenças entre os tratamentos para inibir o escurecimento e tempo de armazenamento para o sabor característico e estranho e gosto amargo (Tabela 3). O sabor característico dos tubérculos minimamente processados decresceu no decorrer do armazenamento. Os tubérculos tratados com metabissulfito de sódio a $0,2 \%$ mantiveram o sabor característico até o décimo dia de armazenamento. $\mathrm{O}$ gosto amargo dos tubérculos já foi percebido no quinto dia e o sabor estranho somente no décimo dia de armazenamento. Até o quinto dia de armazenamento, os tratamentos metabissulfito de sódio a $0,2 \%$, metabissulfito de sódio a $0,1 \%$ associado ao ácido ascórbico a $1 \%$ e branqueamento associado ao metabissulfito de sódio a $0,1 \%$ com vácuo foram aqueles que mantiveram o gosto amargo imperceptível, entre fraco e regular, fato essencial para a aceitação do produto pelo consumidor.

O sabor típico de batata cozida é relativamente fraco, quando comparado com outras hortaliças, e depende da interação de compostos voláteis e solúveis dos constituintes celulares (Taylor et al., 2007). Já foram identificados mais 
de 140 compostos voláteis que contribuem para a formação do sabor típico de batata cozida (Ulrich et al., 2000). As substâncias químicas responsáveis pelo gosto amargo são compostos orgânicos, tais como os alcalóides (Queiroz \& Treptow, 2006). Em batata, o sabor que causa sensação semelhante à ardência se deve a presença do alcalóide solanina, formada pela exposição dos tubérculos à luz. Os sabores estranhos também podem resultar do manejo inadequado das

Tabela 2. Notas para tamanho, formato, defeitos, cor e qualidade geral de tubérculos submetidos a tratamentos de processamento mínimo e armazenados a $5 \pm 1^{\circ} \mathrm{C}$ por 15 dias (notes for size, shape, defects, color and overall quality of potato tubers submitted to minimal processing treatments and stored at $5+1^{\circ} \mathrm{C}$ during 15 days). Santa Maria, UFSM, 2010.

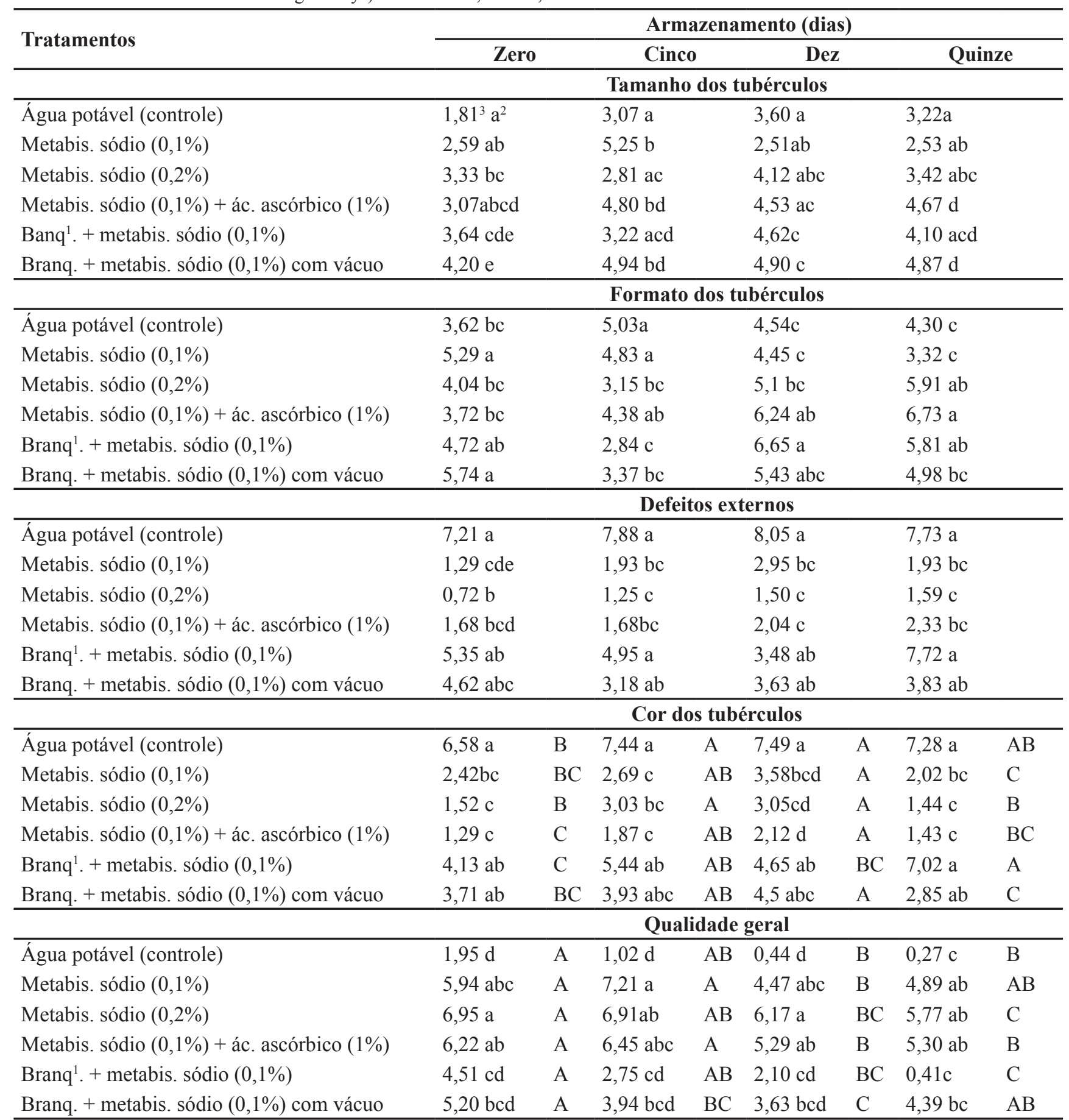

${ }^{1}$ Branqueamento em água aquecida a $80^{\circ} \mathrm{C}$ (proporção de $1 \mathrm{~kg}$ de amostra para $2 \mathrm{~L}$ de água) por 5 min [blanching in water at $80^{\circ} \mathrm{C}$ (ratio of $1 \mathrm{~kg}$ of sample in $2 \mathrm{~L}$ of water) during $5 \mathrm{~min}$ ]; ${ }^{2}$ Tratamentos seguidos por letras diferentes, minúscula na coluna e maiúscula na linha, diferem entre si pelo método de comparações múltiplas de Kruskal-Wallis $(p=0,05)$ [treatments followed by different letters in lowercase in the column and uppercase in the row, differ by Kruskal-Wallis $(p=0.05)$ multiple comparison test]; ${ }^{3}$ Notas de 0 a 9 para o atributo avaliado; Quanto maior a nota em cada item avaliado, melhor a qualidade do atributo aparência (grades 0-9 to the evaluated attributes; The higher the score on each item rated, the better the appearance attribute). 
Tabela 3. Notas para sabor característico e estranho e gosto amargo de tubérculos de batatas submetidos a tratamentos de processamento mínimo, armazenados a $5 \pm 1^{\circ} \mathrm{C}$ por 15 dias e submetidos ao cozimento [grades for flavor, strange and bitter taste of potato tubers submitted to minimal processing treatments and stored at $5+1{ }^{\circ} \mathrm{C}$ for 15 days and cooked]. Santa Maria, UFSM, 2010.

\begin{tabular}{|c|c|c|c|c|c|c|c|c|}
\hline \multirow{2}{*}{ Tratamentos } & \multicolumn{8}{|c|}{ Armazenamento (dias) } \\
\hline & \multicolumn{2}{|c|}{ Zero } & \multicolumn{2}{|c|}{ Cinco } & \multicolumn{2}{|c|}{ Dez } & \multicolumn{2}{|c|}{ Quinze } \\
\hline & \multicolumn{8}{|c|}{ Sabor característico } \\
\hline Água potável (controle) & $4,38^{3} \mathrm{~d}^{2}$ & $\mathrm{~A}$ & $2,81 \mathrm{~d}$ & $\mathrm{AB}$ & $2,75 \mathrm{~cd}$ & $\mathrm{BC}$ & $0,00 \mathrm{~d}$ & $\mathrm{C}$ \\
\hline Metabis. sódio $(0,1 \%)$ & $5,02 \mathrm{bcd}$ & A & $5,04 \mathrm{ab}$ & A & $1,78 \mathrm{~d}$ & $\mathrm{~B}$ & $2,00 \mathrm{bcd}$ & $\mathrm{B}$ \\
\hline Metabis. sódio $(0,2 \%)$ & $4,68 \mathrm{~cd}$ & $\mathrm{AB}$ & $4,63 \mathrm{abc}$ & $\mathrm{BC}$ & $5,78 \mathrm{a}$ & $\mathrm{AB}$ & $2,65 \mathrm{abc}$ & $\mathrm{C}$ \\
\hline Metabis. sódio $(0,1 \%)+$ ác. ascórbico $(1 \%)$ & $5,79 \mathrm{ab}$ & A & $5,29 \mathrm{a}$ & $\mathrm{AB}$ & $4,88 \mathrm{ab}$ & $\mathrm{BC}$ & $3,07 \mathrm{abc}$ & $\mathrm{C}$ \\
\hline Branq $^{1} .+$ metabis. sódio $(0,1 \%)$ & $5,53 \mathrm{abc}$ & A & $4,43 \mathrm{bcd}$ & $\mathrm{AB}$ & $3,33 \mathrm{bcd}$ & $\mathrm{BC}$ & $0,24 \mathrm{~cd}$ & $\mathrm{C}$ \\
\hline \multirow[t]{2}{*}{ Branq. + metabis. sódio $(0,1 \%)$ com vácuo } & $6,00 \mathrm{a}$ & A & $4,14 \mathrm{~cd}$ & $\mathrm{~B}$ & $4,12 \mathrm{abc}$ & $\mathrm{B}$ & $3,44 \mathrm{a}$ & $\mathrm{B}$ \\
\hline & \multicolumn{8}{|c|}{ Sabor estranho } \\
\hline Água potável (controle) & $2,60 \mathrm{a}$ & $\mathrm{AB}$ & $2,11 \mathrm{a}$ & $\mathrm{A}$ & $4,91 \mathrm{a}$ & $\mathrm{BC}$ & $7,25 \mathrm{a}$ & $\mathrm{C}$ \\
\hline Metabis. sódio $(0,1 \%)$ & $0,81 \mathrm{ab}$ & A & $0,54 \mathrm{bc}$ & A & $2,91 \mathrm{~b}$ & $\mathrm{~B}$ & $2,75 \mathrm{~cd}$ & $\mathrm{~B}$ \\
\hline Metabis. sódio $(0,2 \%)$ & $0,22 \mathrm{~b}$ & A & $1,00 \mathrm{ab}$ & $\mathrm{AB}$ & $2,72 b$ & $\mathrm{BC}$ & $3,09 \mathrm{bc}$ & $\mathrm{C}$ \\
\hline Metabis. sódio $(0,1 \%)+$ ác. ascórbico $(1 \%)$ & $0,25 \mathrm{~b}$ & A & $0,73 \mathrm{c}$ & A & $4,91 \mathrm{a}$ & $\mathrm{B}$ & $3,2 \mathrm{bc}$ & $\mathrm{B}$ \\
\hline Branq $^{1} .+$ metabis. sódio $(0,1 \%)$ & $0,40 \mathrm{~b}$ & A & $0,35 \mathrm{c}$ & A & $5,02 \mathrm{a}$ & $\mathrm{B}$ & $6,21 \mathrm{abc}$ & $\mathrm{B}$ \\
\hline \multirow[t]{2}{*}{ Branq. + metabis. sódio $(0,1 \%)$ com vácuo } & $0,26 \mathrm{~b}$ & A & $0,41 \mathrm{c}$ & $\mathrm{AB}$ & $4,33 \mathrm{ab}$ & $\mathrm{C}$ & $2,93 \mathrm{c}$ & $\mathrm{BC}$ \\
\hline & \multicolumn{8}{|c|}{ Gosto amargo } \\
\hline Água potável (controle) & $1,88 \mathrm{ab}$ & $\mathrm{A}$ & $4,62 \mathrm{a}$ & $\mathrm{B}$ & $4,58 \mathrm{a}$ & $\mathrm{B}$ & $6,44 \mathrm{a}$ & $\mathrm{C}$ \\
\hline Metabis. sódio $(0,1 \%)$ & $2,19 \mathrm{ab}$ & A & $3,45 \mathrm{ab}$ & $\mathrm{B}$ & $3,53 \mathrm{bcd}$ & $\mathrm{B}$ & $3,85 \mathrm{abc}$ & $\mathrm{B}$ \\
\hline Metabis. sódio $(0,2 \%)$ & $2,62 \mathrm{a}$ & $\mathrm{BC}$ & $0,81 \mathrm{c}$ & $\mathrm{AB}$ & $1,65 \mathrm{~d}$ & $\mathrm{AB}$ & $3,40 \mathrm{bcd}$ & $\mathrm{C}$ \\
\hline Metabis. sódio $(0,1 \%)+$ ác. ascórbico $(1 \%)$ & $0,62 \mathrm{c}$ & A & $0,50 \mathrm{c}$ & A & $4,10 \mathrm{ab}$ & $\mathrm{B}$ & $3,05 \mathrm{~cd}$ & $\mathrm{AB}$ \\
\hline Branq $^{1} .+$ metabis. sódio $(0,1 \%)$ & $0,83 \mathrm{c}$ & A & $3,78 \mathrm{ab}$ & $\mathrm{B}$ & $3,89 \mathrm{abc}$ & $\mathrm{B}$ & $4,31 \mathrm{abc}$ & $\mathrm{B}$ \\
\hline Branq. + metabis. sódio $(0,1 \%)$ com vácuo & $1,10 \mathrm{bc}$ & A & $1,83 \mathrm{bc}$ & $\mathrm{AB}$ & $2,49 \mathrm{~cd}$ & $\mathrm{BC}$ & $2,91 \mathrm{~d}$ & $\mathrm{C}$ \\
\hline
\end{tabular}

${ }^{1}$ Branqueamento em água aquecida a $80^{\circ} \mathrm{C}$ (proporção de $1 \mathrm{~kg}$ de amostra para $2 \mathrm{~L}$ de água) por 5 min [blanching in water heated at $80^{\circ} \mathrm{C}$ (ratio of $1 \mathrm{~kg}$ of sample in $2 \mathrm{~L}$ of water) during $5 \mathrm{~min}$ ]; ${ }^{2}$ Tratamentos seguidos por letras distintas, minúscula na coluna e maiúscula na linha, diferem entre si pelo método de comparações múltiplas de Kruskal-Wallis $(p=0,05)$ [treatments followed by different letters in lowercase in the column and uppercase in the row, differ by Kruskal-Wallis $(\mathrm{p}=0.05)$ multiple comparison test]; ${ }^{3}$ Notas de 0 a 9 para o atributo avaliado. Quanto maior a nota do sabor característico e menor nota para sabor estranho e gosto amargo, melhor a qualidade do atributo sabor [grades from 0-9 to the evaluated attributes. The higher the note of the characteristic flavor and lowest score for flavor and bitter taste, the better the flavor attribute].

plantas, como o excesso de defensivos e fertilizantes químicos, e dos tubérculos durante a colheita e o armazenamento, como altas temperaturas e falta de ventilação (Friedman, 1997).

A avaliação do atributo dureza dos tubérculos minimamente processados acusou diferenças entre os tratamentos (Tabela 4). A dureza na primeira mordida variou entre regular e moderada, com valores de 3,05 a 6,37. A cremosidade durante a mastigação variou de pouco cremosa até cremosidade moderada, ou seja, entre os valores de 2,45 a 6,07 . A umidade (suculência) variou de pouca a regular em todos os tratamentos, com valores entre 3,09 e 5,59. O aquecimento dos tubérculos, necessário para o branqueamento, resultou na formação de uma fina camada mais densa na superfície, devido à gelatinização do amido, e na perda de líquidos e da cremosidade durante o armazenamento. A textura de um tecido é mantida pelo turgor das células, que é perdido à medida que ocorre o aquecimento. Durante o processo de aquecimento ocorre o inchamento (gelatinização) do grão de amido e a degradação da pectina que forma a lamela média, o que enfraquece as ligações intercelulares (Ormerod et al., 2002). Portanto, o aquecimento ou a cocção dos tubérculos de batata causa o amaciamento dos tecidos e facilita a separação das células. Neste estudo ocorreu o acúmulo de líquidos na embalagem nos tratamentos com branqueamento, que no final do período de armazenamento podem ter resultado em sabor estranho. No entanto, sabor estranho também foi observado no controle, sem o acúmulo de líquido na embalagem.

Os tubérculos de batata minimamente processados apresentaram boa aceitabilidade até o quinto dia de armazenamento, com exceção do tratamento com metabissulfito de sódio a $0,2 \%$ que apresentou até o décimo dia (Tabela 5). Os resultados da intenção de compra expressam as variações observadas nos atributos aparência, sabor e textura, ou seja, todos os atributos avaliados apresentaram valores intermediários $\mathrm{e}$ aceitáveis. Além disso, entre os atributos de sabor, o gosto amargo foi perceptível também no quinto dia, o que corrobora os resultados de aceitabilidade. Tam- 
Tabela 4. Dureza na primeira mordida, cremosidade durante a mastigação e umidade de tubérculos de batatas submetidos a diferentes tratamentos de processamento mínimo, armazenados a $5 \pm 1{ }^{\circ} \mathrm{C}$ por 15 dias e submetidos ao cozimento [hardness at first bite, creaminess and moisture during chewing of potato tubers submitted to different minimal processing treatments and, stored at $5+1{ }^{\circ} \mathrm{C}$ for 15 days and cooked]. Santa Maria, UFSM, 2010.

\begin{tabular}{|c|c|c|c|c|c|c|c|c|}
\hline \multirow{2}{*}{ Tratamentos } & \multicolumn{8}{|c|}{ Armazenamento (dias) } \\
\hline & \multicolumn{2}{|c|}{ Zero } & \multicolumn{2}{|c|}{ Cinco } & \multicolumn{2}{|c|}{ Dez } & \multicolumn{2}{|c|}{ Quinze } \\
\hline & \multicolumn{8}{|c|}{ Dureza na primeira mordida } \\
\hline Água potável (controle) & $6,12^{3} \mathrm{a}^{2}$ & $\mathrm{~A}$ & $4,39 \mathrm{~b}$ & $\mathrm{AB}$ & $3,48 \mathrm{~cd}$ & $\mathrm{BC}$ & $3,12 \mathrm{c}$ & $\mathrm{C}$ \\
\hline Metabis. sódio $(0,1 \%)$ & $4,84 \mathrm{bc}$ & $\mathrm{A}$ & $3,95 \mathrm{~b}$ & $\mathrm{BC}$ & $4,38 \mathrm{bcd}$ & $\mathrm{AB}$ & $3,59 \mathrm{bc}$ & $\mathrm{C}$ \\
\hline Metabis. sódio $(0,2 \%)$ & $4,57 \mathrm{bc}$ & $\mathrm{A}$ & $4,28 \mathrm{~b}$ & A & $3,05 \mathrm{~d}$ & $\mathrm{~B}$ & $4,68 \mathrm{ab}$ & A \\
\hline Metabis. sódio $(0,1 \%)+$ ác. ascórbico $(1 \%)$ & $4,27 \mathrm{c}$ & $\mathrm{BC}$ & $4,71 \mathrm{ab}$ & $\mathrm{AB}$ & $4,83 \mathrm{abc}$ & $\mathrm{A}$ & $3,50 \mathrm{bc}$ & $\mathrm{C}$ \\
\hline Branq $^{1} .+$ metabis. sódio $(0,1 \%)$ & $5,72 \mathrm{ab}$ & $\mathrm{B}$ & $5,80 \mathrm{a}$ & $\mathrm{AB}$ & $6,37 \mathrm{a}$ & $\mathrm{A}$ & $5,23 \mathrm{a}$ & $\mathrm{B}$ \\
\hline \multirow[t]{2}{*}{ Branq. + metabis. sódio $(0,1 \%)$ com vácuo } & $5,25 \mathrm{ab}$ & $\mathrm{BC}$ & $6,22 \mathrm{a}$ & $\mathrm{A}$ & $5,92 \mathrm{ab}$ & $\mathrm{AB}$ & $4,87 \mathrm{a}$ & $\mathrm{C}$ \\
\hline & \multicolumn{8}{|c|}{ Cremosidade durante a mastigação } \\
\hline Água potável (controle) & $3,64 \mathrm{c}$ & $\mathrm{AB}$ & $3,50 \mathrm{bc}$ & $\mathrm{A}$ & $5,81 \mathrm{a}$ & $\mathrm{C}$ & $4,62 \mathrm{a}$ & $\mathrm{BC}$ \\
\hline Metabis. sódio $(0,1 \%)$ & $4,38 \mathrm{bc}$ & $\mathrm{BC}$ & $3,68 \mathrm{bc}$ & A & $5,38 \mathrm{a}$ & $\mathrm{C}$ & $3,93 \mathrm{ab}$ & $\mathrm{AB}$ \\
\hline Metabis. sódio $(0,2 \%)$ & $3,90 \mathrm{c}$ & $\mathrm{A}$ & $4,29 \mathrm{ab}$ & $\mathrm{AB}$ & $5,11 \mathrm{ab}$ & $\mathrm{B}$ & $3,80 \mathrm{abc}$ & A \\
\hline Metabis. sódio $(0,1 \%)+$ ác. ascórbico $(1 \%)$ & $5,04 \mathrm{ab}$ & $\mathrm{BC}$ & $4,70 \mathrm{a}$ & $\mathrm{AB}$ & $6,07 \mathrm{a}$ & $\mathrm{C}$ & $4,15 \mathrm{a}$ & A \\
\hline Branq $^{1} .+$ metabis. sódio $(0,1 \%)$ & $4,83 \mathrm{ab}$ & $\mathrm{C}$ & $3,33 \mathrm{c}$ & $\mathrm{BC}$ & $2,93 \mathrm{~b}$ & $\mathrm{AB}$ & $2,45 \mathrm{c}$ & A \\
\hline \multirow[t]{2}{*}{ Branq. + metabis. sódio $(0,1 \%)$ com vácuo } & $5,31 \mathrm{a}$ & $\mathrm{B}$ & $3,02 \mathrm{c}$ & $\mathrm{A}$ & $2,97 \mathrm{~b}$ & $\mathrm{~A}$ & $2,77 \mathrm{bc}$ & $\mathrm{A}$ \\
\hline & \multicolumn{8}{|c|}{ Umidade } \\
\hline Água potável (controle) & $3,33 \mathrm{~b}$ & $\mathrm{~A}$ & $2,60 \mathrm{c}$ & $\mathrm{A}$ & $5,30 \mathrm{ab}$ & $\mathrm{B}$ & $5,21 \mathrm{a}$ & $\mathrm{B}$ \\
\hline Metabis. sódio $(0,1 \%)$ & $3,59 \mathrm{~b}$ & $\mathrm{AB}$ & $3,09 \mathrm{c}$ & A & $5,59 \mathrm{a}$ & $\mathrm{C}$ & $4,44 \mathrm{abc}$ & $\mathrm{BC}$ \\
\hline Metabis. sódio $(0,2 \%)$ & $3,87 \mathrm{~b}$ & $\mathrm{~A}$ & $3,88 \mathrm{abc}$ & $\mathrm{A}$ & $4,40 \mathrm{c}$ & $\mathrm{B}$ & $4,24 \mathrm{bc}$ & $\mathrm{AB}$ \\
\hline Metabis. sódio $(0,1 \%)+$ ác. ascórbico $(1 \%)$ & $4,83 \mathrm{a}$ & $\mathrm{AB}$ & $3,98 \mathrm{ab}$ & $\mathrm{A}$ & $5,51 \mathrm{a}$ & $\mathrm{C}$ & $5,08 \mathrm{ab}$ & $\mathrm{BC}$ \\
\hline Branq $^{1} .+$ metabis. sódio $(0,1 \%)$ & $4,20 \mathrm{ab}$ & $\mathrm{AB}$ & $4,42 \mathrm{a}$ & $\mathrm{B}$ & $4,67 \mathrm{bc}$ & $\mathrm{B}$ & $3,72 \mathrm{c}$ & A \\
\hline Branq. + metabis. sódio $(0,1 \%)$ com vácuo & $5,04 \mathrm{a}$ & B & $3,45 \mathrm{bc}$ & $\mathrm{B}$ & $4,95 \mathrm{abc}$ & B & $3,91 \mathrm{c}$ & A \\
\hline
\end{tabular}

${ }^{1}$ Branqueamento em água aquecida a $80^{\circ} \mathrm{C}$ (proporção de $1 \mathrm{~kg}$ de amostra para $2 \mathrm{~L}$ de água) por 5 min [blanching in water heated to $80^{\circ} \mathrm{C}$ (ratio of $1 \mathrm{~kg}$ of sample in 2 L of water) during $5 \mathrm{~min}$ ]; ${ }^{2}$ Tratamentos seguidos por letras diferentes, minúscula na coluna e maiúscula na linha, diferem entre si pelo método de comparações múltiplas de Kruskal-Wallis $(p=0,05)$ [treatments followed by different letters in lowercase in the column and uppercase in the row, differ by Kruskal-Wallis $(\mathrm{p}=0.05)$ multiple comparison test]; ${ }^{3}$ Notas de 0 a 9 para $o$ atributo avaliado. Quanto maior a nota em cada item avaliado, melhor a qualidade do atributo textura [grades from 0-9 for the evaluated attributes. The higher the score on each item rated, the better the texture attribute].

Tabela 5. Notas de intenção de compra dos tubérculos de batatas submetidos a diferentes tratamentos de processamento mínimo e armazenados a $5 \pm 1^{\circ} \mathrm{C}$ por 15 dias [grades of the intention to purchase potato tubers submitted to different minimal processing treatments and stored at $5 \pm 1^{\circ} \mathrm{C}$ during 15 days]. Santa Maria, UFSM, 2010.

\begin{tabular}{lllllllll}
\hline \multirow{2}{*}{ Tratamentos } & \multicolumn{9}{c}{ Armazenamento (dias) } \\
\cline { 2 - 8 } & \multicolumn{3}{c}{ Zero } & \multicolumn{3}{c}{ Cinco } & \multicolumn{1}{c}{ Dez } & \multicolumn{3}{c}{ Quinze } \\
\hline Água potável (controle) & $3,30^{3} \mathrm{~d}^{2}$ & $\mathrm{~A}$ & $3,34 \mathrm{~d}$ & $\mathrm{~A}$ & $1,18 \mathrm{~d}$ & $\mathrm{~B}$ & $0,19 \mathrm{~d}$ & $\mathrm{~B}$ \\
Metabis. sódio $(0,1 \%)$ & $4,05 \mathrm{~cd}$ & $\mathrm{AB}$ & $4,72 \mathrm{bcd}$ & $\mathrm{A}$ & $3,08 \mathrm{abc}$ & $\mathrm{BC}$ & $1,38 \mathrm{bcd}$ & $\mathrm{C}$ \\
Metabis. sódio $(0,2 \%)$ & $4,59 \mathrm{bcd}$ & $\mathrm{A}$ & $5,00 \mathrm{abc}$ & $\mathrm{A}$ & $4,15 \mathrm{abc}$ & $\mathrm{AB}$ & $1,82 \mathrm{abc}$ & $\mathrm{B}$ \\
Metabis. sódio $(0,1 \%)+$ ác. ascórbico (1\%) & $6,40 \mathrm{a}$ & $\mathrm{A}$ & $6,10 \mathrm{a}$ & $\mathrm{A}$ & $2,64 \mathrm{bcd}$ & $\mathrm{B}$ & $2,69 \mathrm{ab}$ & $\mathrm{B}$ \\
Branq ${ }^{1}+$ + metabis. sódio $(0,1 \%)$ & $4,91 \mathrm{abc}$ & $\mathrm{A}$ & $4,02 \mathrm{~cd}$ & $\mathrm{AB}$ & $1,61 \mathrm{~cd}$ & $\mathrm{BC}$ & $1,07 \mathrm{~cd}$ & $\mathrm{C}$ \\
Branq. + metabis. sódio $(0,1 \%)$ com vácuo & $6,35 \mathrm{ab}$ & $\mathrm{A}$ & $5,54 \mathrm{ab}$ & $\mathrm{AB}$ & $3,70 \mathrm{ab}$ & $\mathrm{BC}$ & $3,17 \mathrm{a}$ & $\mathrm{C}$ \\
\hline
\end{tabular}

${ }^{1}$ Branqueamento em água aquecida a $80^{\circ} \mathrm{C}$ (proporção de $1 \mathrm{~kg}$ de amostra para $2 \mathrm{~L}$ de água) por 5 min [blanching in water heated to $80^{\circ} \mathrm{C}$ (ratio of $1 \mathrm{~kg}$ of sample in $2 \mathrm{~L}$ of water) during $5 \mathrm{~min}$ ]; ${ }^{2}$ Tratamentos seguidos por letras diferentes, minúscula na coluna e maiúscula na linha, diferem entre si pelo método de comparações múltiplas de Kruskal-Wallis $(p=0,05)$ [treatments followed by different letters in lowercase in the column and uppercase in the row, differ by Kruskal-Wallis $(p=0.05)$ multiple comparison test]; ${ }^{3}$ Notas de 0 a 9 para $o$ atributo avaliado. Quanto maior a nota, melhor a intenção de compra [grades from 0-9 for the evaluated attributes. The higher the score, the better the buying intention]. 
bém, odores desagradáveis surgem pela presença de processos anaeróbicos, comprometendo a aceitação do produto (Pineli et al., 2005a). Tubérculos de batata da cultivar Ágata embaladas a vácuo parcial e armazenados a $5^{\circ} \mathrm{C}$ mantiveram a firmeza, porém apresentaram a superfície enrugada, a embalagem encharcada e leve odor desagradável a partir do sexto dia de armazenamento, relacionados a processos fermentativos indesejáveis (Pineli et al., 2005b).

A qualidade sensorial é o conjunto de características capazes de impressionar nossos sentidos. $\mathrm{O}$ ato de consumir um alimento é resultante de uma série de atitudes frente ao produto (Queiroz \& Treptow, 2006). A vida de prateleira do produto pode ser determinada pelo tempo requerido para que a intenção de compra atinja determinada aceitabilidade (Ares et al., 2008). Assim, os tubérculos de batata minimamente processados apresentaram uma boa intenção de compra até o quinto dia de armazenamento, o que pode ser considerado adequado para a matéria-prima processada e a tecnologia utilizada. Apesar de representar vantagens ao consumidor e ganho econômico aos produtores pela agregação de valor, o processamento mínimo diminui a vida de prateleira, devido aos danos físicos provocados pelas operações de preparo, que alteram a taxa respiratória e a evolução do etileno, deterioram a textura e aumentam a produção de compostos indesejáveis (Chitarra, 2001).

Os resultados deste trabalho mostram que é possível aumentar o aproveitamento dos tubérculos de batata, mesmo sendo de uma mistura de cultivares, com tamanhos fora dos padrões exigidos para o comércio in natura e, portanto, de baixo valor comercial para o mercado fresco. Tubérculos uniformes com diâmetro entre 30 e $45 \mathrm{~mm}$ podem ser utilizados para a elaboração de produtos minimamente processados de alto valor agregado e com vida de prateleira compatível com os padrões da Europa, que varia entre 5 e 7 dias (Jaouen, 2006). O domínio do processo de elaboração possibilita aumentar a qualidade e o valor agregado dos produtos e, em consequência, o retorno econômico e a sustentabilidade da cadeia produtiva da batata. Entretanto, a padronização dos tubérculos facilita o manuseio em grandes quantidades, minimiza as perdas e aumenta a produção da indústria e a qualidade do produto (Chitarra \& Chitarra, 2005), principalmente para os atributos aparência, sabor e textura. A aplicação do branqueamento elimina os microorganismos contaminantes, porém altera a cor dos tubérculos e afeta a aceitação do produto pelos consumidores. Portanto, tubérculos de batata com diâmetro entre 30 e $45 \mathrm{~mm}$, minimamente processados, têm boa aceitação até o quinto dia de armazenamento a $5^{\circ} \mathrm{C}$. No entanto, a manutenção da vida de prateleira depende da aplicação de metabissulfito de sódio.

\section{AGRADECIMENTOS}

À Fundação de Amparo à Pesquisa do estado do Rio Grande do Sul, pela concessão de bolsa de iniciação científica, e ao Conselho Nacional de Desenvolvimento Científico e Tecnológico, pela concessão de bolsas e financiamento parcial da pesquisa.

\section{REFERÊNCIAS}

AHVENAINEN R. 1996. New approaches in improving the shelf life of minimally processed fruit and vegetables. Trends in Food Science and Technology 7: 179-187.

ALENCAR SM; KOBLITZ MGB. Oxirredutases. In: KOBLITZ MGB. (coord). Bioquímica de Alimentos. Rio de Janeiro, Guanabara Koogan, 2008. p. 125-152.

AOAC. 2005. Official methods of analysis of the Association of Official Analytical Chemists International. $18^{\text {th }}$ ed. Gaithersburg: AOAC n.p.

ARAÚJO JMA. 2010. Química de alimentos: teoria e prática. 4. Ed. Viçosa: UFV. 596 p.

ARES G; MARTÍNEZ I; LAREO C; LEMA P. 2008. Failure criteria based on consumers' rejection to determine the sensory shelf life of minimally processed lettuce. Poshavest Biology and Technology 49: 255-259.

CABEZAS-SERRANO A; AMODIO ML; CORNACCHIA R; RINALDI R; COLELLI G. 2009. Suitability of five different potato cultivars (Solanum tuberosum L.) to be processed as fresh-cut products. Postharvest Biology and Technology 53: 138-144.

CHITARRA MIF; CHITARRA AD. 2005. Póscolheita de frutas e hortaliças: fisiologia e manejo. Lavras: UFLA. 785 p.

FRIEDMAN M. 1997. Chemistry biochemistry, and dietary role of potato polyphenols. Journal of Agricultural and Food Chemistry
45: 1523-1540.

GÓMEZ-LÓPEZ VM; RAJKOVICA; RAGAERT P; SMIGICN; DEVLIEGHERE F. 2009. Chlorine dioxide for minimally processed produce preservation: a review. Trends in Food Science \& Technology 20: 17-26.

GRAY D; HUGHES JC. 1978. Tuber quality. In: The potato crop, the scientific basis for improvement (Ed. PM Harris). London: Chapman Hall, p. 504-544.

GREGORY JF. 2010. Vitaminas. In: DAMODARAN S; PRKIN KL; FENNEMA OR (ed) Química de alimentos de Fennema. 4.ed. Porto Alegre: Artemed. p. 346-404.

HAASE NU. 2008. Healthy aspects of potatoes as part of the human diet. Potato Research 51: 239-258.

INSTITUTO ADOLFO LUTZ. 2009. Métodos físico-químicos para análise de alimentos. Normas Analíticas Instituto Adolfo Lutz. 1. ed. eletrônica. Disponível em: http://www. ial.sp.gov.br/.

JACOMINO AP; ARRUDA MC; BRON IU; KLUGE RA. 2008. Transformações bioquímicas em hortícolas após a colheita. In: KOBLITZ MGB (coord) Bioquímica de alimentos. Rio de Janeiro: Guanabara Koogan. p. 153-189.

JACQUES-SILVA MC; NOGUEIRA CW; BROCH LC; FLORES EMM; ROCHA JBT. 2001. Diphenyl diselenide and ascorbic acid changes deposition of selenium and ascorbic acid in liver and brain of mice. Pharmacology \& Toxicology 88: 119-125.

JAOUEN N. 2006. Packaging ideas for freshcut products. In: Fresh-cut Europe: The new European fresh-cut conference. Disponível em: http://www.freshcuteurope.com/.

LANTE A; ZOCCA F. 2010. Effect of $\beta$-cyclodextrin addition on quality of precooked vacuum packed potatoes. Food Science and Technology 43: 409-414.

LAURILA E; KERVINEN R; AHVENAINEN R.1998. The inhibition of enzymatic browning in minimally processed vegetables and fruits. Postharvest News and information 9: 53-66.

MORETTI CL. 2007. Panorama de processamento mínimo de frutas e hortaliças. In: Manual de processamento mínimo de frutas e hortaliças. Brasília: Embrapa Hortaliças e SEBRAE. p. 25-40.

ORMERO DA; RALF SJ; JOBLING A; GIDLEY M. 2002. The influence of starch swelling on the material properties of cooked potatoes. Journal of Materials Science 37: 1667-1673.

PINELI LLO; MORETTI CL; ALMEIDA GC; NASCIMENTO ABG; ONUKI ACA. 2005a. Associação de atmosfera modificada e antioxidantes reduz o escurecimento de batatas "Ágata" minimamente processadas. Horticultura Brasileira 23: 993-999.

PINELI LLO; MORETTI CL; ALMEIDA GC; ONUKI ACA; NASCIMENTO ABG. 2005b. Caracterização química e física de batatas 'Agata' minimamente processadas embaladas sob diferentes atmosferas ativas. Pesquisa Agropecuária Brasileira 40: 1035-1041.

QUEIROZ MI; TREPTOW RO. 2006. Análise sensorial para a avaliação da qualidade dos 
alimentos. Rio Grande: FURG. 268 p.

ROCCULI P; GALINDO FG; MENDONZA F; WADSÖ L; ROMANI S; DALLA ROSA M; SJÖHOLM I. 2007. Effects of the application of anti-browning substances on the metabolic activity and sugar composition of freshcut potatoes. Phostharvest Biology and Technology 43: 151-157.

ROCHA AMCN; COULON EC; MORAIS AMMB. 2003. Effects of vacuum packaging on the physical quality of minimally processed potatoes. Food Service Technology 3: 81-88.

SAEG. 2007. Sistema para análises estatísticas. Versão 9.1: Viçosa: UFV. Fundação Arthur Bernardes.

TAYLOR MA; MCDOUGALL GJ; STEWART D. 2007. Potato flavor and texture. In: VREUGDENHIL D (ed) Potato biology and biotechnology: advances and perspectives. Elsevier. p. 525-540.

THYBO AK; KAACK JCK; PETERSEN MA. 2006. Effect of cultivars, wound healing and storage on sensory quality and chemical components in pre-peeled potatoes'. Food Science and Technology 39: 166-176.

TUDELA JA; SPIN JC; GIL MI. 2002. Vitamin $\mathrm{C}$ retention in fresh-cut potatoes. Postharvest Biology and Technology 26: 75-84.

ULRICH D; HOBERG E; NEUGEBAUER H; TIEMANN H; DARSOW U. 2000. Investigation of the boiled potato flavor by human sensory and instrumental methods. American Journal of Potato Research 77: 111-117. 